\title{
Consumer-friendly Shopping Assistance by Personal Behavior Log Analysis on Ubiquitous Shop Space
}

\author{
Somkiat SAE-UENG, Sineenard PINYAPONG, Akihiro OGINO, Toshikazu KATO
}

Chuo University, Science and Engineering 1-13-27 Kasuga Bunkyo-ku Tokyo 112-8551 JAPAN,

\{somkiat, sineenard, ogino, kato\}@indsys.chuo-u.ac.jp

\begin{abstract}
This paper has proposed a shopping assistance service. We provide consumer-friendly services which based on personal behavior log data. The consumer-friendly services are information service which display through a monitor, robot, sound and music that can attract new consumer. In this paper, we study personal behavior from a log file that has been recorded by RFID and camera sensors in the ubiquitous environment. Utilizing ubiquitous sensors, we apply three types of observation scopes to model each user's preference. They are microscopic, mezzoscopic, and macroscopic observation scopes. We have proposed the behavior analysis, algorithms and collaborative filtering to retrieve information. Finally, we will also propose a ubiquitous shop space experiment.
\end{abstract}

Key words: Recommendation Services, Shopping Assistance, Shopping Behavior, Ubiquitous Computing

\section{Introduction}

There is growing interest in using ubiquitous computing technologies to build systems that support our daily lives more effectively. If the environment surrounding us could recognize our activities indirectly by sensors, the novel services, which respect our activities, would be possible. Examples of such systems are smart offices, classrooms, and homes that allow computers to monitor what is happening in the environment, to figure out how to most effectively assist in the tasks being performed, and to have sufficient output devices to assist effectively.

For example, a classroom might help students take more effective notes by capturing much of the details of a lecture, freeing the student to pay more attention to the content of the class and simply annotating or indexing into the record of the experience for later review. A meeting room could record the events and discussion in a meeting to allow others who did not attend the meeting to efficiently find out what happened. An office could remind its user of relevant information, or signal urgent messages. A machine room could provide documentation for operators or visiting repair technicians. A home could babysit children or allow the elderly to remain self-sufficient. A building could supplement the capabilities of physically or mentally challenged individuals through visual assistance, movement assistance such as opening and closing doors, and providing and receiving information in alternate forms such as lip-reading and sign-language recognition. These systems can be built into the environment as well as being hand-held or even worn as part of our clothing. Thinking thoroughly, we will find that one of the most important factors for such systems is the recognition of human intentions by using ubiquitous sensors. This means various data collection methods of the system (or the computer) rely on sensor and human before processing those information into different presentation.

This research paper will mainly focus on recommending the products to the customers. The study showed that recommending a product to the customer required collecting a customer profile, purchasing history, and customer's preferences. Then these data would be filtered to process a product to be recommended. This stage of research could be seen in many current research projects. However, according to the study, we understood that to effectively recommend a product, the right presentation toward the right customer is required; therefore, we would propose consumer-friendly shopping assistance by personal behavior log analysis on ubiquitous shop space. We review in section 2, our personal behavior and their log. In sections 3, we describe our shopping assistance framework. In section 4, implementation and experiment are described. This is 
followed by conclusions with directions for future work (section 5).

\section{Personal Behavior and Their Log \\ 2.1 Behavior pattern}

From the study of consumer behavior, when consumers need a product they will search for the desired one by observing, screening and comparing the available information. In case of apparel, there is also a need for a trial. The definition of consumer behavior in our study has been defined as followings.

Table 1. Definitions for Behaviors Pattern towards Items.

\begin{tabular}{|c|l|}
\hline Actions & \multicolumn{1}{c|}{ Definition } \\
\hline Standing & $\begin{array}{l}\text { The state in which a consumer stop by at } \\
\text { a certain distance in front of a certain } \\
\text { object. }\end{array}$ \\
\hline Viewing & $\begin{array}{l}\text { The state in which a consumer looks at a } \\
\text { certain object. }\end{array}$ \\
\hline Touching & $\begin{array}{l}\text { The state in which a consumer touches a } \\
\text { certain object where the object does not } \\
\text { separate from its shelf. }\end{array}$ \\
\hline Carrying & $\begin{array}{l}\text { The state in which a consumer separately } \\
\text { picks up a certain object from its shelf. }\end{array}$ \\
\hline Fitting & $\begin{array}{l}\text { The state in which a consumer uses a } \\
\text { mirror to match a certain object with } \\
\text { his/her appearance. }\end{array}$ \\
\hline
\end{tabular}

\subsection{Why are these five actions important?}

Human beings possess fives organs which perform the sense of awareness. They are eyes, ears, nose, tongue and the body. These organs are functioning for seeing, listening, smelling, tasting and touching. Though human beings were born with the same five senses of feelings, they have dissimilar interest. This research has observed the behavior of consumers. When they have interest in a product, they will stare at it or pick it up to take a glance. The time spent for taking a closer look at the product depends on his/her interest. Occasionally, they might go back to review it due to a sudden interest in it.

This paper has focused on a shopping, mainly on an apparel shop. The breakdown of measurement and summary results has been constructed by five actions standing, viewing, touching, carrying and fitting.

\subsection{How to detect five actions?}

Under Ubiquitous environment, our system will use sensors which consist of RFID and Camera which act as an observer and memory for details of consumers. In other words, RFID will perform in checking who the consumer is. The good point of RFID is when it is located in the length of 40-50 Centimeter of detecting signal; it will be able to detect the signal and to tell what type of consumer they are. Cameras are installed extensively in the shop and different shelves in order to store the consumer behaviors.

In addition, we also notice the consumer behavior by using three dissimilar positions of cameras. They comprise a wide capture (to view the position of consumer), a panning capture (to view the movement position of a consumer) and a close-up capture (to view the detailed shot of what product a consumer is picking up). This observation method and use of sensor under ubiquitous environment will help collect various behaviors of consumers. After that, a further analysis and the product recommendation towards the right consumers with the matching needs can be made effectively.

\subsection{Evaluation}

In a shopping space, consumers usually spend more time looking at items for information or product, which they like, rather than those they do not. Thus, viewing time, and behavior towards items, may provide a means of identifying consumer behavior, or propensity to consume a product or information service.

We assume the time that consumers spent in front of goods on the shelf may be a good predictor. We also assume that consumers will touch items in which they have an interest. We hypothesize that a consumer is interested in an item when he touches and stays in front of the item for a length of time.

In the system process, we gather the consumer behavior, there must be a data summary so that we can use that summary for the following analysis (Table 2).

Table 2. Definitions for Evaluations of Actions toward Items

\begin{tabular}{|c|l|}
\hline Evaluations & \multicolumn{1}{c|}{ Definition } \\
\hline Period & $\begin{array}{l}\text { The time that a consumer spends on a } \\
\text { behavior measured in seconds. }\end{array}$ \\
\hline Frequency & $\begin{array}{l}\text { The number of times that a consumer } \\
\text { has a behavior. }\end{array}$ \\
\hline
\end{tabular}

\section{Shopping Assistance Framework}

\subsection{Consumer-Friendly Service}

A basic thinking tells us that human beings have different preference therefore the recommendation of information with similar content to consumers can be presented in non-identical media. In this paper, we have presented information to consumers in three methods as follows. 


\section{Display}

The study reveals that most consumers prefer to purchase online because of the availability of product features and the presentation of related products which also cover the comparison of specification. This enrichment of information stimulates a quick purchasing decision. In contrast, if the consumers go to buy a product at the regular shop, they will merely watch the product without the availability of product details. Although there might be some information given by the salesperson, the complete product details do not avail. Nevertheless, some consumers dislike to buy online since they cannot touch and actually view the product.

As a result of that, this research brought in the strong points of those two ways of buying, which are to be able to view the actual product and to be able to compare the similar features of product, to become visible simultaneously. To do so, a display monitor will be placed next to a product. When a consumer picks up that product, other items in the same product category as well as each product features and the shelf location will be shown.

\section{Robot}

The 21st century is the genuinely technology era and a robot has gradually become an integral part of our daily life, for example, AIBO, ASHIMO, etc. To recommend a product via a Robot is another alternative that can attract the interest of consumers. Besides, the robot can interact with the consumers as well.

\section{Sound and Music}

Many research papers disclosed that when the consumers shop in the store where there is light music playing in the background, they would feel relaxed and spend longer shopping time [9]. This research paper has proposed the recommendation of information via the sound of music. While consumers are choosing the goods, they can also listen to the sound of other product recommendations at the same time.

\subsection{Augmented Space}

We have been developing our shop space which we call augmented space in ubiquitous environment. The augmented space is composed of microscopic and macroscopic view devices. We can describe their as follows:

\section{Microscopic View Devices}

Each shelf is equipped with (a) an RFID tag reader to identify each consumer around the area, The RFID tag reader can read the member IDs and the information recorded in RFID tags. (b) several item cameras to detect their behaviors, related to the items, such as touching, grasping and wearing, and (c) several LCD monitors to show personal messages to them, as well as to show public messages to the consumers.

\section{Macroscopic View Devices}

The ceiling of the shop is equipped with a camera array to cover the entire area, without occlusion, to detect the location of each consumer at each time slice.

\subsection{System Process}

\subsubsection{Personal Behavior Log}

A behavior log analysis method of consumer behavioral analysis, under ubiquitous environment, is a method which has sensors (RFID, camera) installed to study the consumer's interest and selection process. The consumer behavioral observation can be categorized as followings.

\section{Observation Methods}

Utilizing ubiquitous sensors, we apply three types of observation methods which are Macroscopic, Microscopic, and Mezzoscopic view as shown in Fig.1 to model each user's preference.

- Macroscopic view

Macroscopic view covers the location of each person in a space by a matrix of global view cameras located on the ceiling. It also covers the overall spatial allocation and density of the people as well as the items in the space.

- Microscopic view

Microscopic view identifies a user from his/her RFID nametag and some handling motions on some item by locally equipped cameras.

- Mezzoscopic view

Mezzoscopic view extracts and traces each person's location and behavior as a time series by using the data from microscopic and macroscopic views.

- Indirect Interaction in Active Observation

The enforcement of answering a huge number of questionnaires on users is a bottleneck in modeling personal preferences. One idea is just taking their behavior log via ubiquitous sensors, without asking them, and mining some specific features by statistical analysis. Such a method is called passive observation. From the aspect of a ubiquitous information environment, future store projects [21], for instance, proposes a smart shelf to monitor their goods with RFID tags in real-time, communicating information with the store server as well as a consumer's shopping cart. The basic idea still 


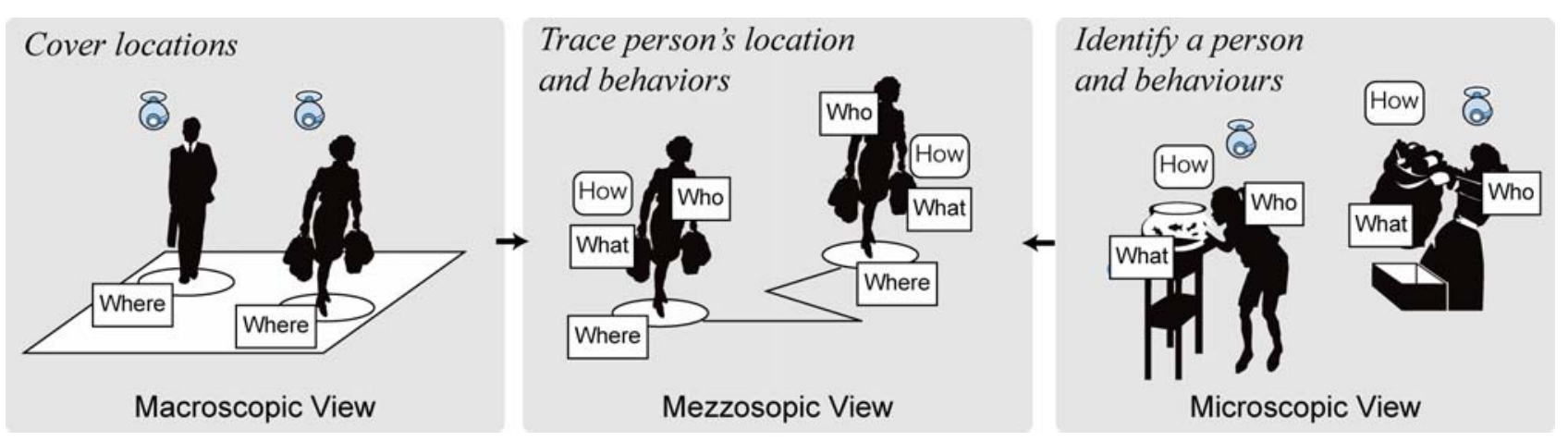

Fig. 1. Macroscopic, Microscopic and Mezzoscopic Views

remains in a passive observation scheme while it refers personal preference data of each consumer. The problem of this method is that it requires long time and huge personal log to cover enough behavior data.

Our idea is to show several messages to each user, i.e., applying active observation, without expecting direct answers. If a message is informative and interesting to a user, he may pay attention, gaze, and follow the suggestion according to the message. In this process he is freely behaving by his intention without feeling any enforcement to answer to the system. In this case, monitoring each user's behavior, i.e., responses to the messages, via ubiquitous sensors enables to attain enough behavior data effectively. This method corresponds to indirect interaction in active observation.

\subsubsection{Personal Behavior Log Analysis}

From those three methods of consumer behavioral observation, we have collected the information in a log file then transferred it into an analysis. The analysis part can be broken down based on the recommendation to the consumers as followings:

Table 3. Personal Behavior and presentation devices

\begin{tabular}{|l|l|}
\hline \multicolumn{1}{|c|}{ Personal Behavior } & $\begin{array}{c}\text { Selected Presentation } \\
\text { Devices }\end{array}$ \\
\hline Consumer looks at display & Display \\
\hline $\begin{array}{l}\text { Consumer looks at robot } \\
\begin{array}{l}\text { Consumer pays attention to } \\
\text { product item }\end{array}\end{array}$ & Robot \\
\hline
\end{tabular}

\section{Algorithm}

The algorithm for recommending service by personal behavior log analysis is as following.

1. It checks the place of a consumer by Macroscopic device and identifies the consumer by Microscopic device.

2. It accumulates data on the consumer's actions towards items with his/her identity and information on these items using Microscopic devices.

3. It finds common features of items, in which this consumer showed interest, by analyzing features of accumulated data.

4. It recommends personalized item information to the consumer, based on his/her behavior.

5. It collects data on the consumer's reaction towards a recommended item in order to find further item features used to improve recommending service.

\subsubsection{Information Filtering}

Most previously proposed recommendation systems that use collaborative filtering can cause problems when there are insufficient user ratings. We introduce our approach to automatic rating recommendation of products by concentrating on collaborative filtering.

Collaborative filtering, the recommendation for a consumer is based on his similarity in terms of ratings with other consumers that constitute what we shall call their neighborhood.

Generally, Collaborative filtering requires a consumer to rate items they are interested in (previous consumer ratings). However, our shop space will arrange the rating of consumers automatically from the pattern of action behavior. It's unnecessary to have consumers fill in the questionnaire or input the ratings themselves. The system can guess the closet needs of consumers.

Table 4. Personal Behavior Log and Automatically Rating by level of interest (rating scale range 1-5, 1= lowest, 5 = highest)

\begin{tabular}{|l|c|}
\hline \multicolumn{1}{|c|}{ Personal Behavior } & $\begin{array}{c}\text { Consumer's level of } \\
\text { interest in product }\end{array}$ \\
\hline $\begin{array}{l}\text { Purchase } \\
\text { (reactlow the Recommendation }\end{array}$ & 5 \\
\hline Fitting (Test a product) & 4 \\
\hline Touch a product & 3 \\
\hline Pause to look at the product & 2 \\
\hline
\end{tabular}


Those mentioned above have to fall within the specified time range. This experiment has set the time limit within 5 second. In case of pause to look at the product and touch a product action, if it's less than the limited range, the system will assume that a consumer simply window-shopped and not assigned rating value. After that, this observation will be used for the recommendation.

In order to create recommendations for the current member, This system performs three steps:

- It compares the current user's ratings against every other member's ratings. Collaborative filtering computes a similarity value for every other member.

- Based on the ratings of the most similar members (neighbors), it predicts the rating the current member would give to every item she has not yet rated.

- It suggests to the member the items with highest predicted rating.

\section{Implementation and Experiment}

\subsection{Implementation}

For the experiment, we have built a 30-square meter room to be simulated as Ubiquitous Smart Space. Six-items-unit display shelves were installed. Each shelf carried an information display (EIZO FlaxScan L365), speaker, and robot on the top level, so that the information of subjects presented could be easily seen on the screen when they selected items, and four pieces of clothes, different in color and type, on the lower levels.

Four cameras (Panasonic Network Camera BL-C31) were used for each shelf to monitor hand motions of the subjects. The other camera placed on the ceiling was used to monitor location of the subject in the shop space. The Fedora Core Linux operating system was stored with a log file and used for showing item information in the shop space. Moreover, RFID units (OMRON V720 Series) were used to identify subjects if they approached the front of the shelf. Sixty-four pieces of clothes were randomly selected to be placed on the shelves throughout the experiment.
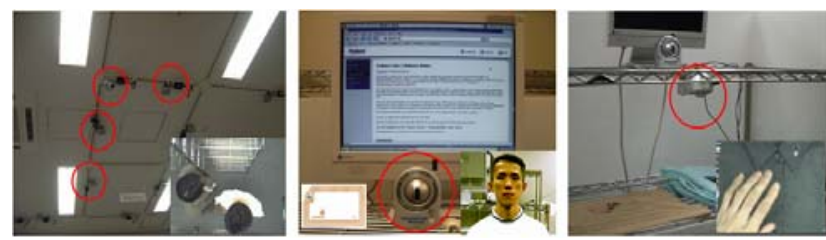

Fig. 2. System Overview of Ubiquitous Shop Space.

\subsection{Experiment}

This experiment has performed by examinees having their actions observed while purchasing items of interest in the ubiquitous shop space. The experiment was conducted by using 18 examinees as consumers. The examinees came to the shop independently three times and bought the clothes in which they were interested. The examinee could feel free to move around in the store.

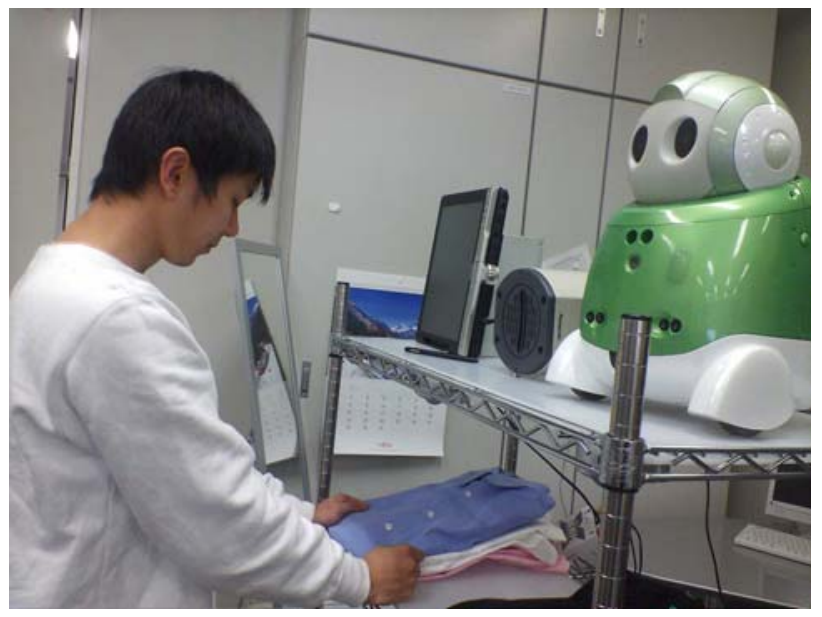

Fig. 3. Examinee, Display, Speaker, and Robot in Ubiquitous Shop Space

The ubiquitous shop space system registered the identity of each examinee in the consumer database before starting the experiment. In the first experiment, the system made an individual model of each examinee by observing their actions towards clothes, and analyzing common features of the clothes in which they showed interest though their actions. In the second and third experiment, the ubiquitous shop space recommended personalized clothing items through information on a display, and it accumulated data on consumers' passive reactions to the recommended information. Clothes items in the shop were replaced after the first and second experiment.

The recommended information about clothes was decided by the personal behavior model for each examinee, which was made in the first experiment. The system showed the recommendation information about clothes, to the information display in the front of the examinee, when it observed consumer identity or presence by microscopic and macroscopic devices. Questionnaires were distributed to each examinee to ask about satisfaction of personalized recommendations. In a successful case, the examinees moved to a place in the store on the basis of their personalized recommendation. 
On average about $60 \%$ of examinees reacted to their personalized recommendation. The results of experiment are shown in Fig. 4.

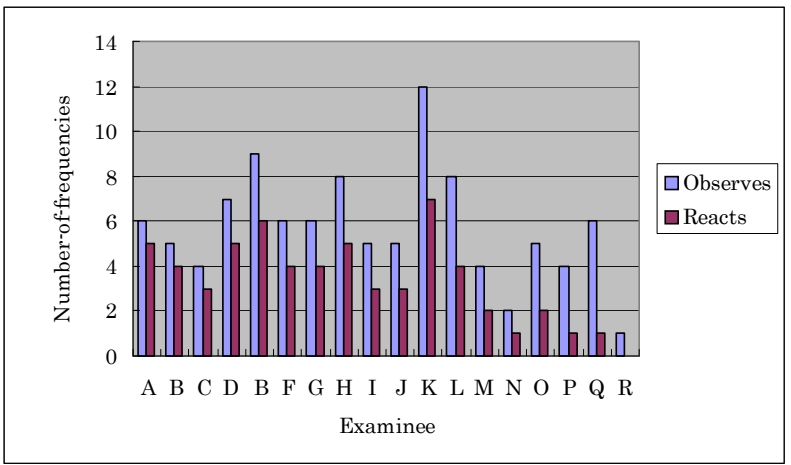

Fig. 4. Frequencies which each Examinee Observes and Reacts to Recommended Personalized Information

\section{Conclusion}

This paper has proposed consumer-friendly shopping assistance services based on personal behavior log data on ubiquitous shop space. The consumer-friendly services are information service which display through a monitor, robot, sound and music available for new consumers. In this paper, we study a personal behavior from log file that has been recorded by RFID and camera sensors in the ubiquitous environment. Utilizing ubiquitous sensors, we apply three types of observation scopes to model each user's preference. They are microscopic, mezzoscopic, and macroscopic observation scopes. We have proposed behavior analysis, algorithms and filtering to retrieve information. Finally, we have also proposed a ubiquitous shop space experiment.

Our system can provide suitable and controlled messages to users to build up their precise preference model without placing any stress on them. Thus, the system can statistically analyze the degree of interest on objects by each person's behavior and can effectively track their behaviors without having them explicitly answer a questionnaire. Therefore, a handful of advanced technologies, such as speech recognition, communication robot, etc will be necessarily developed in the future. Afterwards the consumer-friendly assistance services will be better fit the consumer.

\section{Acknowledgement}

This research is partially supported by Grant-in-Aid for Scientific Research (S), No. 19100004, "Robotics modeling of diversity of multiple KANSEI and situation understanding in real space", Japan Society for the
Promotion of Science.

\section{References}

1. P. Chatterjee, D.L. Hoffman, and T. P. Novak, Modeling the Clickstream: Implications for Web-based Advertising Efforts, Rutgers University, 1998.

2. C. Dennis, D. Marsland, T. Cockett and V. Hlupic, "Market segmentation and customer knowledge for shopping center”, 25th Int. Conf. Information Technology Interfaces (ITI2003), pp417-424, 2003. Journal for Numerical Methods in Engineering 1991; 36(5):765-781.

3. K. Higuchi, et al., Modeling KANSEI through Real World Interaction with Ubiquitous Information Environment -Smart Sphere and Smart Store, Proc. of 6th Asia Design Conference, Oct. 2003.

4. Sung Young Jung, Jeong-Hee Hong, Taek-Soo Kim, "A Statistical Model for User Preference," IEEE Transactions on Knowledge and Data Engineering, vol. 17, no. 6, pp.834-843, June 2005.

5. T. Kato, "Modeling Individualities of Personal Preferences and Situations via Mobile and Ubiquitous Environment - KANSEI Engineering for Personal Assistance Service”, invited talk, HC'2006,Aizu, Sep 7th, 2006.

6. J. Krumm, S. Harris, B. Meyers, B. Brumitt, M. Hale and S. Shafer, "Multi-Camera Multi-Person Tracking for Easy Living," Proc. of 3rd IEEE International Workshop on Visual Surveillance, pp.3-10, 2000.

7. J.H. Lee, G. Appenzeller, H. Hashimoto, "Physical Agent for Sensored Networked and Thinking Space,” Proc. of the 1998 IEEE International Conference on Robotics \& Automation, pp838-843, 1998.

8. T. Mori, K. Asaki, H. Nogushi, T. Sato: "Accumulation and summarization of Human Daily Action Data in One-Room-Type Sensing System” . IROS2001, pp2349-2354 Oct. 2001.

9. Ronald E. Milliman, "Using Background Music to Affect the Behavior of Supermarket Shoppers", Journal of Marketing, Vol. 46, No. 3 (Summer, 1982), pp. 86-91

10. National Institute of Information and Communications Technology Keihanna Human Info Communications Research Center Distributed and Cooperative Media Group the UKARI Project Website:http://univ.nict.go.jp/past_pj/disco/eng/resea rch/ukari_project.html 
11. M. Pantic and L. J. M. Rothkrantz, "Toward an Affect-Sensitive Multimodal Human-Computer Interaction”, Proceedings of the IEEE. Vol.91, No.9, pp.1370-1390, 2003.

12. Hyunjung Park, Jeehyong Lee, “A Framework of Context-Awareness for Ubiquitous Computing Middlewares,” icis, pp. 369-374, Fourth Annual ACIS International Conference on Computer and Information Science (ICIS'05), 2005.

13. A. Pentland, R. Picard and P. Maes "Smart Rooms, Desks, and Clothes: Toward Seamlessly Networked Living,'British Telecommunications Engineering, Vol.15, pp.168-172, July, 1996.

14. S. Pinyapong and T. Kato, "A Framework of Time, Place, Purpose and Personal Profile Based Recommendation Service for Mobile Environment,” IEICE Trans. Inf. \& Syst., vol.E88-D, no.5, pp.937-946, May 2005.

15. S. Sae-ueng, Akihiro Ogino and T. Kato, "Modeling Personal Preference Using Shopping Behaviors in Ubiqitous Information Environment”, DEWS2007, Mar. 2007.

16. N. A. Streitz, "From Individual Work and
Desktop-Based Collaboration to Ubiquitous Meeting Environments, Multimedia, Hypermedia, and Virtual Reality: Models, Systems, and Applications, 1996.

17. T. Terada, M. Tsukamoto, S. Nishio, “Active GIS: A Geographic Information System Using Active Database Systems," Proc. of Symposium on ASIA GIS 2001 (CD-ROM), June 2001.

18. P. Underhill, Why We Buy; The Science of Shopping. Simon \& Schuster, Touchstone Book, 1999, 2000.

19. M. Weiser, "The Computer for the Twenty-First Century,”Scientific American, pp.94-104, September 1991.

20. Daisuke Yamamoto, Miwako Doi, Nobuto Matsuhira, Hirotada Ueda, Masatsugu Kidode, Behavior Fusion in a Robotic Interface for Practicality and Familiarity -Approach by Simultaneous Imitations-. 13th IEEE International Workshop on Robot and Human Interactive Communication, Sep. 2004.

21. The METRO Group Future Store Initiative; http://www.future-store.org

22. Web Site Personalization and Collaborative Filtering; http://www.vogoo-api.com 